\title{
Tratamento cirúrgico de isquemia mesentérica por rotação de raiz mesentérica espontânea - Relato de caso
}

\author{
Surgical Treatment of Spontaneous Mesenteric Root Rotation Mesenteric Ischemia - Case \\ Report
}
Tratamiento quirúrgico de la isquemia mesentérica espontánea de rotación de la raíz mesentérica - Reporte de un caso

Marcos André Costa Freire ${ }^{1 *}$, Izabela Augusta de Oliveira Medeiros ${ }^{2}$, Elaine Francisca Coimbra de Araújo', Julia Costa Justo ${ }^{3}$, Isadora Gomes Mesquita ${ }^{3}$, Larissa Pessoa de Oliveira ${ }^{3}$, Thomás Benevides Said ${ }^{3}$, Felipe Machado Pinheiro Leão ${ }^{3}$, Juan Eduardo Rios Rodriguez ${ }^{3}$.

\section{RESUMO}

Objetivo: Relatar um caso de isquemia mesentérica aguda por rotação de raiz mesentérica espontânea sem sinais de embolia e discutir sobre as bases do diagnóstico e a importância do manejo precoce. Relato do caso: paciente do sexo masculino, 57 anos, deu entrada na urgência cirúrgica com quadro de distensão e dor abdominal há 1 dia associada a parada de eliminação de fezes e flatos. A radiografia de abdome evidenciou distensão importante de alças de delgado e presença de níveis hidroaéreos. Este foi internado para realização de laparotomia exploradora de urgência. Foi feita enterectomia, $50 \mathrm{~cm}$ após o ângulo de Treitz até $3 \mathrm{~cm}$ do cólon ascendente, com sinais de isquemia. Considerações Finais: $O$ melhor método para minimizar as consequências e a taxa de morbidade é o diagnóstico precoce. Logo, deve-se buscar o aprimoramento clínico e cirúrgico tanto no pré quanto no pós-operatório a fim de obter prognóstico melhor desses pacientes.

Palavras-chave: Isquemia mesentérica, Laparotomia, Cirurgia geral.

\begin{abstract}
Objective: To report a case of acute mesenteric ischemia due to spontaneous mesenteric root rotation without signs of embolism and discuss the basis of the diagnosis and the importance of early management. Case report: A 57-year-old male patient was admitted to the emergency room with distension and abdominal pain for 1 day associated with stool and flatus elimination. Abdominal radiography showed significant distension of the slender loops and presence of airborne levels. He was hospitalized for emergency exploratory laparotomy. Enterectomy was performed $50 \mathrm{~cm}$ after the Treitz angle up to $3 \mathrm{~cm}$ from the ascending colon, with signs of ischemia. Final Considerations: The best method to minimize consequences and morbidity rate is early diagnosis. Therefore, clinical and surgical improvement should be sought both preoperatively and postoperatively in order to obtain a better prognosis of these patients.
\end{abstract}

Keywords: Mesenteric ischemia, Laparotomy, General surgery.

\section{RESUMEN}

Objetivo: Informe un caso de isquemia mesentérica aguda debido a la rotación espontánea de la raíz mesentérica sin signos de embolia y discuta la base del diagnóstico y la importancia del tratamiento temprano.

\footnotetext{
${ }^{1}$ Hospital Universitário Getúlio Vargas (HUGV), Manaus - AM *E-mail: mandrefreire@hotmail.com

2 Instituto de Cirurgia do Estado do Amazonas (ICEA), Manaus - AM

${ }^{3}$ Universidade Federal do Amazonas (UFAM), Manaus - AM
} 
Relato del caso: Informe del caso: un paciente masculino de 57 años ingresó en la sala de emergencias con distensión y dolor abdominal durante un día, asociado con la interrupción de la eliminación de heces y flatos. La radiografía abdominal mostró una distensión significativa de los bucles delgados y la presencia de niveles en el aire. Fue hospitalizado por una laparotomía exploratoria de emergencia. La enterectomía se realizó 50 $\mathrm{cm}$ después del ángulo de Treitz hasta $3 \mathrm{~cm}$ desde el colon ascendente, con signos de isquemia. Considerações finais: El mejor método para minimizar las consecuencias y la tasa de morbilidad es el diagnóstico temprano. Por lo tanto, se debe buscar una mejoría clínica y quirúrgica tanto preoperatoria como posoperatoria para obtener un mejor pronóstico de estos pacientes.

Palavras-clave: Isquemia mesentérica, Laparotomía, Cirugía general.

\section{INTRODUÇÃO}

O sistema vascular esplâncnico, responsável por manter a irrigação arterial intestinal é formado pelas seguintes estruturas - tronco celíaco (TC), artéria mesentérica superior (AMS) e artéria mesentérica inferior (AMI), que por sua vez, esses mesmos vasos estão conectados entre si por outros vasos, formando uma grande rede vascular de anastomoses que são capazes de manter o suprimento arterial intestinal adequado podendo desenvolver vias colaterais a fim manter o sistema vascular intestinal compensado. A AMS é responsável pela irrigação do segmento intestinal até os $2 / 3$ distais do colón, já a AMI supri o terço distal do colón transverso até o reto proximal (CLAIR DG, 2016)

Quando, por certos fatores, acontece uma insuficiência vascular esplâncnica, ou seja, a rede de anastomoses não consegue suprir a necessitado do órgão com o fluxo sanguíneo adequado, dá-se o nome a essa síndrome de isquemia mesentérica. Tal patologia é rara, acometendo aproximadamente 2.000 pessoas a cada ano na Inglaterra, contra os aproximadamente 100.000 casos de infarto agudo do miocárdio, porém possui uma taxa de mortalidade que chega aos $70 \%$. Uma pesquisa realizada nos Estados Unidos entre 1998 e 2010 revelou um decréscimo do número de casos de 9,6 por 100.000 para 6,7 por 100.000 e atualmente avalia-se que apenas $1 \%$ dos pacientes internados com abdômen agudo seja devido a isquemia mesentérica aguda (IMA), representando 0,1\% das internações hospitalares (BARROS MJ, 2018)

Identificou-se que síndrome isquêmica atinge em maiores proporções indivíduos com idade maior que 50 anos, mulheres em uma razão de 3:1, são identificados alguns fatores de risco como: doença cardíaca valvular ou aterosclerótica, arritmias cardíacas, infarto agudo do miocárdio, insuficiência cardíaca descompensada e hipovolemia e/ou hipotensão (KASSAHUN WT et al, 2008). A mesma pode ser classificada em relação a diferentes aspectos, são eles - fluxo, podendo ser oclusiva ou não oclusiva, quanto a sintomatologia podendo ser aguda ou crônica e quanto a origem, se é arterial ou venoso - e suas principais causas são embolia arterial que é associado, geralmente, a trombos murais associados a infarto agudo do miocárdio ou fibrilação atrial, trombose arterial, mais comum em pacientes idosos e com aterosclerose e por isquemia não oclusiva podendo está relacionado a pacientes cardiopatas (GOULART PA et al, 2014)

O quadro clínico é inespecífico e a dor intensa e de início súbito e desproporcional é o marcador mais precoce, principalmente na região umbilical, porém pode ser no epigástrio. O paciente pode ter discreta taquicardia, está inquieto e sudoreico, com o passar do tempo, sinais peritonite como sensibilidade, defesa e rigidez abdominal começam a surgir, sugerindo então o desenvolvimento de necrose nas alças intestinais. Independentemente da etiologia, a mesentérica superior é a artéria mais comumente atingida, representando $67 \%$ dos casos de IMA (ALONSO NEP, 2012)

O diagnóstico precoce é de extrema importância, pois à medida que o infarto intestinal ocorre, a mortalidade aumento significativamente, sendo o diagnóstico clínico a maneira mais importante de realizá-lo, prioriza-se a história patológica pregressa do paciente, principalmente se houve alguma patologia cardíaca associada como fibrilação atrial ou patologia valvular. Caso o diagnóstico não esteja claro, são necessários exames complementares como hemograma, angiografia mesentérica ou tomografia computadorizada angiográfica que apresenta alta sensibilidade e especificidade (CLAIR DG, 2016) 
O tratamento consiste em primeiro estabilizar o paciente com reposição volêmica e analgesia acompanhadas de monitoramento contínuo. Discute-se ainda a implementação de antibioticoterapia a fim de evitar e ampliar focos de infecção. Institui-se posteriormente a revascularização mesentérica, seja por via endovascular ou por cirurgia convencional. Entre as principais complicações da IMA cita-se: peritonite, sepse e necrose intestinal, sendo necessária no último a ressecção cirúrgica da região intestinal acometida. (CUI J, 2018)

O objetivo foi relatar um caso de isquemia mesentérica aguda por rotação de raiz mesentérica espontânea sem sinais de embolia e discutir sobre as bases do diagnóstico e a importância do manejo precoce identificado em um Hospital Pronto Socorro da cidade de Manaus. Trata-se de patologia rara principalmente em pacientes homens na idade adulta.

\section{DETALHAMENTO DO CASO}

Paciente D.G.F, sexo masculino, 57 anos, deu entrada na urgência cirúrgica com quadro de distensão e dor abdominal há 1 dia associada a parada de eliminação de fezes e flatos. Ao exame físico apresentava-se com queda importante do estado geral, taquidispnéico, hipocorado, desidratado, abdome distendido, ruído hidroaéreo ausente, doloroso a palpação difusamente, descompressão brusca positiva em todos os quadrantes com sinais de peritonite. Radiografia de abdômen evidenciando distensão importante de alças de delgado e presença de níveis hidroaéreos.

Este foi internado para realização de laparotomia exploradora de urgência devido a peritonite, foi realizada incisão xifopubica, a fim de ter uma boa observação da cavidade, foi achado grande quantidade de secreção sero-hemática pútrida livre na cavidade, rotação anômala de alças intestinais fazendo constrição da raiz do mesentério. Constatou-se a presença de divertículo de Merckel há $15 \mathrm{~cm}$ do ângulo de Treitz, isquemia extensa de delgado, estendendo-se há $50 \mathrm{~cm}$ do ângulo de Treitz até cólon ascendente. Após esses achados foi realizada enterectomia com isolamento e ligadura dos vasos do mesentério na área acometida (Figura 1) e optado por reconstrução em segundo tempo cirúrgico.

Figura 1 - Produto da enterectomia, após o ângulo de Treitz até $3 \mathrm{~cm}$ do cólon ascendente, com sinais de isquemia.

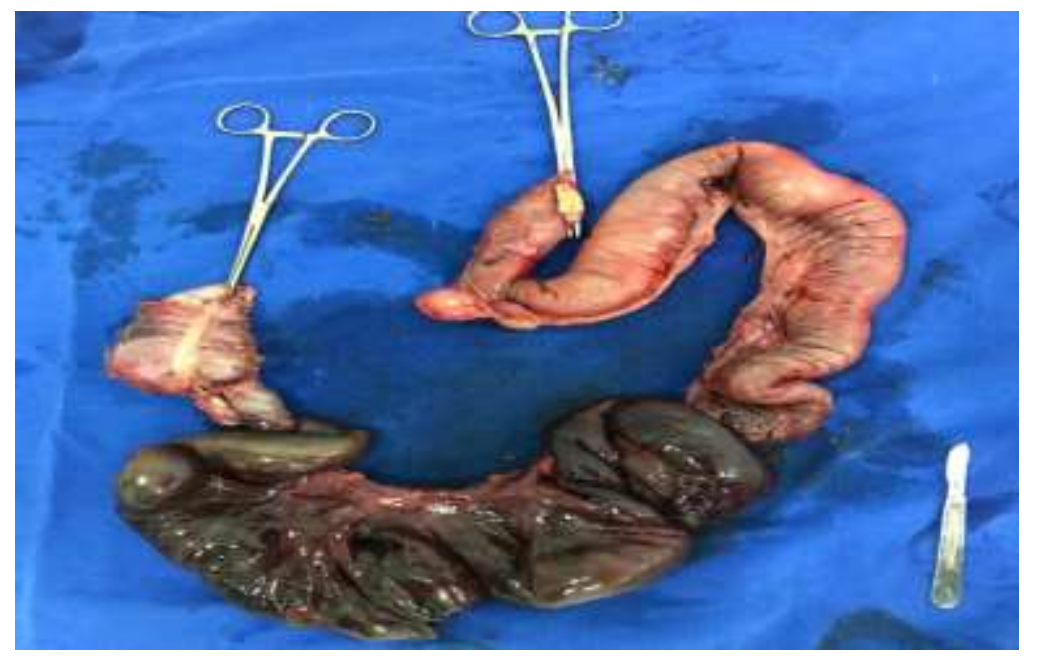

Fonte: Freire MAC, et al., 2019.

Após o procedimento cirúrgico o paciente permaneceu sedado, entubado e sem droga vasoativa na Unidade de Terapia Intensiva. Foi iniciado dieta parenteral, no $3^{\circ}$ dia de internação submetido à relaparotomia exploradora com enteroanastomose jejuno-transverso término-terminal, em plano único, com intercorrência de pneumotórax hipertensivo por acidente de punção de subclávia direita. 
Ao exame físico paciente encontrava-se sedado com escala de RASS de sedação de 3 , estável hemodinamicamente, bem perfundido, tórax assimétrico com enfisema subcutâneo em hemitórax esquerdo, Frequência Cardíaca:75 batimentos por minuto; Pressão arterial: 100x60; Saturação de Oxigênio: 95\%.

Durante $\circ 6^{\circ}$ dia de internação o paciente apresentou-se grave, porém hemodinamicamente estável sem o uso de aminas vasoativas, sob o efeito de sedoanalgesia com fentanil, bem acoplado a prótese ventilatória, abre os olhos ao chamado e tenta contactar. Além disso encontrava-se anictérico, acianótico, hipocorado $(1+/ 4)$, afebril e com permanência de enfisema subcutâneo no hemitórax esquerdo. Teve evolução lenta, apresentando intercorrências de injuria renal aguda e pneumonia relacionada à assistência à saúde. Já no $21^{\circ}$ dia encontrava-se em regular estado geral; emagrecido; afebril; acianótico; sem sedação; responsivo e respirando espontaneamente em ar ambiente, obtendo alta no $25^{\circ}$ dia de internação.

\section{DISCUSSÃO}

Apresentamos um caso de isquemia mesentérica aguda, o caso citado encaixa-se em dentro do grupo de abdome agudo vascular e trata-se de um paciente adulto sem doença cardíaca prévia e sem sinais de tromboembolia com admissão em um Pronto Socorro (PS), na cidade de Manaus, Amazonas, (FERES O e PARRA RS, 2008). A isquemia mesentérica é uma patologia que pode ser tanto um quadro agudo, como um crônico. Sua forma aguda é rara, acomete principalmente a artéria mesentérica superior, porém possui uma alta taxa de mortalidade chegando a $70 \%$. As principais causas de isquemia mesentérica são embolia arterial que é associado, geralmente, a trombos murais associados a infarto agudo do miocárdio ou fibrilação atrial, trombose arterial, mais comum em pacientes idosos e com aterosclerose e por isquemia não oclusiva podendo estar relacionado a pacientes cardiopatas (GOULART PA et al, 2014).

Uma vez que ocorre queda brusca de fornecimento de oxigênio necessário para suprir as atividades metabólicas do intestino, observa-se, vasodilatação como resposta inicial a isquemia e posteriormente, notase vasoconstrição à medida que a lesão evolui. Por conseguinte, há o acometimento da mucosa e submucosa intestinal, predispondo a translocação de bactérias do lúmen intestinal. Ocorre ampla resposta inflamatória resultando em maior dano às alças intestinais (CLAIR MD, 2016).

A principal etiologia é a embolia arterial, originada, principalmente, de trombos murais oriundos de infarto agudo do miocárdio e fibrilação atrial. Já a isquemia causada por trombose arterial está relacionada à infecção abdominal prévia, hérnia estrangulada, neoplasia ou coagulopatias, sendo também relacionada ao uso de algumas medicações como contraceptivos orais, diuréticos e vasopressores. E a isquemia não oclusiva é menos frequente sendo a vasoconstrição associada à insuficiência cardíaca a principal responsável por esse distúrbio (VIRGINI-MAGALHÃES CEV, 2009).

Cerca de dois terços dos pacientes são do sexo feminino com mais de 70 anos, na anamnese deve-se observar todo e qualquer sinal de doença vascular ou aterosclerótica. Em nosso caso, porém, nota-se que o paciente é do sexo masculino, ou seja, representa um terço dos pacientes admitidos e encontra-se fora da faixa etária de risco sem doenças cardiovasculares prévias, ressaltando a raridade do caso apresentado. Clinicamente, a isquemia mesentérica aguda pode se manifestar de diversas formas, fato que retarda 0 diagnóstico e propicia complicações. Comumente os pacientes evoluem com dor intensa, devido ao vasoespasmo intestinal, seguida de náuseas, vômitos, e sangramento retal, podendo evoluir com irritação peritoneal, distensão abdominal e febre. Ao exame físico, nota-se dor a palpação superficial, diminuição dos ruídos hidroaéreos, sudorese e taquicardia (VIRGINI-MAGALHÃES CEV, 2009).

As alterações laboratoriais mais frequentes são: hemoconcentração, leucocitose, e em casos mais avançados, acidose metabólica, contudo tais distúrbios apresentam pouca especificidade. Logo, devem ser solicitados exames de imagem quando disponíveis como: radiografia de abdômen, tomografia computadorizada, eco-doppler, angioressonância e arteriografia, a qual é o exame padrão-ouro para diagnóstico de isquemia mesentérica aguda e crônica (MARINO MA, et al., 2009).

O tratamento depende da evolução do paciente, condição clínica, comorbidades e principalmente da etiologia da isquemia, prioriza-se a revascularização em até 8 horas após o início dos sintomas em pacientes 
hemodinamicamente estáveis, por via endovascular seja por tromboaspiração, trombólise ou angioplastia com colocação de stents, com o objetivo de evitar a progressão da necrose e proporcionar um tratamento menos agressivo. É considerado o tratamento de primeira escolha uma vez que é menos invasivo e apresenta menores taxas de complicações no peri-operatório, consequentemente menores indicies de morbimortalidade. (SOARES MJBCB, 2018)

Em estágios mais grave, a laparotomia exploradora é preconizada, a fim de avaliar a viabilidade das alças intestinais e extensão da necrose. A parte necrosada é ressecada e embolectomia aberta é a técnica mais utilizada a fim de restituir o fluxo sanguíneo em casos de isquemia da AMS por êmbolos. Já em casos de lesão por aterosclerose utiliza-se o Bypass à AMS ou tromboendarterectomia. No caso relatado, optou-se pela ressecção, uma vez que parte da alça intestinal se encontrava em avançado estado de necrose, no entanto, não foram observados êmbolos ou trombos que justifiquem a isquemia em questão (SOARES MJBCB, 2018). Em alguns casos, a ressecção sem a reperfusão prévia é recomendada, com o objetivo de evitar a síndrome de reperfusão, que pode ser definida como reabsorção de bactérias e de substâncias próinflamatórias devido à revascularização, potencializando respostas sistêmicas e distúrbios hidroeletrolíticos (VIRGINI-MAGALHÃES CEV, 2009).

Em todas as alternativas terapêuticas, geralmente, utiliza-se terapia anticoagulante com heparina IV a fim de evitar novos eventos trombóticos. O pós-operatório segue com monitorização contínua do paciente, reposição volêmica de suporte e analgesia, em casos de peritonite ou choque séptico uma segunda abordagem laparoscópica pode ser necessária em conjunto com uso de drogas vasoativas de suporte da unidade de terapia intensiva (UTI) (ALONSO NEP, 2012).

\section{CONSIDERAÇÕES FINAIS}

Tendo em vista que a isquemia mesentérica apresenta evolução clínica rápida e que apesar de rara, possui uma taxa de mortalidade muito elevada, o diagnóstico precoce torna-se a melhor forma de reduzir as taxas de óbito, e a abordagem cirúrgica permanece como o tratamento padrão da maioria dos casos. Logo, devese ter aprimoramento do suporte clínico tanto no pré quanto no pós-operatório a fim de diagnosticar os pacientes precocemente e minimizar o grau de lesão. Entretanto, alguns detalhes relevantes podem passar despercebidos por alguns profissionais, seja por descuido ou pelos sintomas inespecíficos da doença. Trazendo a nossa região, o Amazonas grande extensão territorial com barreiras geográficas importantes e poucos centros de referência, o que dificulta a mobilidade destes pacientes em caso de queixas menores.

\section{REFERÊNCIAS}

1. ALONSO NEP, ALEMÁN IG. Enfermedad veno-oclusiva inflamatoria mesentérica. Acta Médica del Centro, 2012, 6 (3) 71-76.

2. CLAIR DG, BEACH JM. Mesenteric ischemia. New England Journal Med. 2016, 374(10):959-68.

3. CUI J, KIRKBY B. Intestinal ischemia secondary to superiormesenteric venous thrombosis-A case report. International Journal of Surgery 2018; 53:96-8.

4. FERES O, PARRA RS. Abdômen Agudo. Medicina (Ribeirão Preto) 2008; 41 (4): 430 - 6

5. GOULART PA, et a. Isquemia mesentérica aguda: relato de caso. Revista do Médico Residente, 2014, 16 (1)

6. KASSAHUN WT, et al. Unchanged high mortality rates from acute occlusive intestinal ischemia: six-year review. Langenbecks Arch Surg 2008; 393: 163-71

7. MARINO MA, et al. Intervenções percutâneas na isquemia arterial mesentérica: indicações, técnica e tratamento. Revista Brasileira de Cardiologia Invasiva, 2009, 17 (4), 533-544.

8. PARK WM, et al. Contemporary management of acute mesenteric ischemia: factors associated with survival. Journal of Vascular Surgery 2002; 35: 445-52.

9. SOARES, MJBCB Isquemia Mesentérica - Clínica, Diagnóstico E Tratamento. 2018. Dissertação (Mestrado Integrado Em Medicina) - Instituto De Ciências Biomédicas Abel Salazar, Universidade Do Porto, Porto, 2018

10. VIRGINI-MAGALHÃES CE, MAYALL MR. Isquemia mesentérica. Revista do Hospital Universitário Pedro Ernesto. 2009;8(1):70-80. 